\title{
La coordinación docente en la universidad: análisis de la situación actual
}

\section{Teacher Coordination at University: Analysis of the Current Situation}

\author{
María José Bolarín Martínez 1 \\ mbolarin@um.es \\ Universidad de Murcia, España
}

\section{Resumen:}

Actualmente, en nuestro sistema educativo universitario, se plantea un cambio causado por la implementación del nuevo modelo educativo europeo. Para responder a la lógica de la reforma planteada por el Espacio Europeo de Educación Superior, se hace necesaria una adaptación de los órganos de gobierno, Administración educativa, directivos de los diversos niveles de las universidades, profesorado, estudiantes, personal no docente, la comunidad educativa, en general. Este modelo nos reta, entre otros aspectos, a pasar desde la actual cultura organizativa universitaria caracterizada por el individualismo, en algunos casos el aislamiento profesional y la competitividad, hacia una nueva cultura compartida y asumir nuevas formas de organización del trabajo que requieren la acción conjunta de todos los implicados. Este trabajo tiene como objetivo poner de manifiesto la importancia de la coordinación como uno de los elementos claves en el cual se articulan las nuevas titulaciones para su buen funcionamiento, al amparo de Bolonia y analizar la situación actual en la universidad española.

\begin{abstract}
:
The Bologna process embodies a new model of university education that is bringing changes to the Spanish university system. The adoption of the European Higher Education Area (EHEA) framework calls for adjustments involving education authorities, governance bodies, teaching and nonteaching staff, students and the community and overall society. Challenges raised by that model include the move from a culture of individualism, professional isolation and competition to one of sharing and working together. This paper aims to highlight, on the one hand, the importance of coordination as one of the key elements around which the new degrees should revolve for the purpose of functioning in line with the Bologna guidelines. On the other hand, it seeks to analyse the current situation in Spanish universities.
\end{abstract}

1 Dirección para correspondencia (correspondence address):

María José Bolarín Martínez. Dpto. de Didáctica y Organización Escolar. Facultad de Educación. Universidad de Murcia. Campus de Espinardo. 30100 Murcia (España). 
Palabras claves:

Coordinación; universidad; interdisciplinar; cultura compartida .

\section{Keywords:}

Coordination; university; interdisciplinary; shared culture.

\section{Résumé:}

Actuellement, dans notre système éducatif de l'université, un changement provoqué par la mise en œuvre du nouveau modèle éducatif européen est proposé. Pour répondre à la logique de la réforme proposée par l'Espace européen de l'enseignement supérieur, une adaptation des organes du gouvernement, Administration éducative, directeurs pédagogiques, gestionnaires de différents niveaux des universités, enseignants, étudiants, personnel non enseignant, la communauté éducative en général. Ce modèle nous incite, entre autres choses, à passer de la culture organisationnelle de l'université actuelle caractérisée par l'individualisme, dans certains cas, l'isolement et la compétitivité professionnelle, à une nouvelle culture commune et à assumer de nouvelles formes d'organisation du travail qui nécessitent une action commune de tous les acteurs. Ce document vise à souligner l'importance de la coordination comme l'un des éléments clés qui permet le fonctionnement harmonieux des nouveaux diplômes, en vertu de Bologne et analyse la situation actuelle dans les universités.

\section{Mots clés:}

Coordination; université; culture interdisciplinaire; partagée.

Fecha de recepción: 21-6-2014

Fecha de aceptación: 2-2-2015

\section{Hacia una cultura de trabajo compartida}

En la actualidad la universidad se encuentra en un proceso de cambio y de transformación de la cultura universitaria. Las universidades necesitan adaptarse para dar respuesta a las diversas exigencias de la sociedad (Schein, 1998) y precisan de una adaptación en relación a: la organización de los planes de estudios, las metodologías de enseñanzaaprendizaje, rol del profesorado y del alumnado, funciones que ha de desarrollar, nuevas formas de trabajo y de entender la evaluación de los aprendizajes, etc. Se trata, en definitiva, de convertir los sistemas universitarios europeos en un referente internacional en lo relativo a la calidad de la formación impartida (Calderón y Escalera, 2008) y ello requiere de una transformación organizativa, docente y discente, donde la coordinación aparece como el elemento clave en el cual se articula la nueva estructura universitaria que cada vez más tiende hacia una cultura compartida.

Para comprender la importancia de la coordinación en el nuevo panorama educativo debemos aproximarnos al conocimiento de la Univer- 
sidad entendida como una organización docente conformada por unos ámbitos que interactúan y se interrelacionan en lo que denominamos cultura organizativa. En cada organización y, más concretamente en la Universidad, existe una estructura organizativa formal (ámbito de la estructura); en ella, se desarrollan determinadas relaciones entre los individuos que la forman (ámbito de las relaciones), cada uno de los individuos que la forman tienen sus propias concepciones y creencias acerca de las personas, las relaciones, cómo hacer las cosas, de enfrentarse a las situaciones nuevas (ámbito de las creencias, valores, percepciones del profesorado) y todo este entramado anterior tiene su proyección en el aula, donde el profesor no actúa porqué sí, si no que está condicionado por cada uno de los ámbitos anteriores (ámbito del aula), pero ¿que implicaciones tiene el nuevo modelo educativo en cada uno de estos ámbitos? y, ¿qué papel juega la coordinación en cada uno de ellos?

En primer lugar cabe citar el ámbito de la estructura, que hace referencia a cómo está organizada la Universidad. Constituye, por así decirlo, el "andamiaje" o "esqueleto" (González, 2007) y, es el resultante de la interacción de normativas, regulaciones oficiales, Decretos, leyes, reglas establecidas por el Ministerio y sus adaptaciones a cada Comunidad Autónoma que confieren el armazón de cada Universidad, con una estructura similar pero que a su vez poseen un funcionamiento diferente y nos define cómo están articulados formalmente sus elementos (González, 2007).

Si revisamos la normativa acerca de cómo deben estar estructuradas las Universidades, nos encontramos que en el Real Decreto 1393/2007, de 29 de octubre, por el que se establece la ordenación de las enseñanzas universitarias oficiales, se establece la estructura de las enseñanzas universitarias y se regulan los estudios universitarios oficiales de Grado.

Y, más concretamente es en la Ley Orgánica 6/2001, de 21 de diciembre, de Universidades, modificada el 17 de septiembre de 2014, en el Titulo $V$ artículo 31 dedicado a la Garantía de la Calidad, donde se recoge la necesidad de establecer criterios de garantía de calidad que faciliten la evaluación, certificación y acreditación, y considera la garantía de calidad como un fin esencial de la política universitaria. Asimismo, esta nueva organización de las enseñanzas universitarias, el RD 1393/2007 y, su posterior modificación el RD 861/2010, incorpora la garantía de calidad como uno de los elementos básicos que un plan de estudios debe contemplar. Esa garantía de calidad, a nivel organizativo, exige a 
las universidades una mejora de la calidad a través de la introducción de mecanismos de evaluación internos y externos. Se denomina Sistema de Garantía de Calidad (a partir de ahora SGC) al conjunto de procedimientos documentados necesarios para implantar la Gestión de la Calidad, partiendo de una estructura organizativa y de unos recursos determinados; sería como la herramienta elaborada para asegurar el control, la revisión y mejora continua de los objetivos de las titulaciones; los sistemas de acceso y admisión de estudiantes; la planificación, desarrollo y resultados de las enseñanzas; el personal académico; los recursos materiales y servicios, en cumplimiento con lo indicado en el Real Decreto $861 / 2010$. Para ello las universidades han diseñado el Sistema de Garantía de Calidad, como elemento clave en el control del cumplimiento de los diseños de las nuevas titulaciones, como una condición para el proceso de verificación y acreditación de los Títulos. No se trata aquí de hacer un recorrido histórico sobre los antecedentes normativos del SGC, pero si de contextualizar a nivel organizativo qué implicaciones tiene en la coordinación. Con su implementación se espera responder a la demanda de necesidades y expectativas de todos los grupos de interés, ofreciendo transparencia, incorporando estrategias de mejora continua, y ordenando las actuaciones sistemáticamente para que contribuyan de modo eficaz a la garantía de calidad. En este contexto legal aparecen las figuras de coordinación docente en el SGC como un elemento clave del sistema educativo universitario en el proceso de Seguimiento de los Títulos Oficiales.

Para hacer realidad este nuevo marco educativo debemos asumir un cambio en la estructura que afecta tanto a la gestión académica como a la intervención docente en las aulas. Esa estructura se caracteriza, entre otros aspectos, por su complejidad y, por ende por su división del trabajo en tareas y su asignación a diferentes personas. Dada esta división, se hace necesario ofrecer condiciones y mecanismos para favorecer la coordinación y un funcionamiento conjunto e integrado entre los elementos que la componen y que ocupan diferentes posiciones formales representadas en el organigrama de la institución en la consecución de una meta común.

Todo ello exige, entre otros aspectos, que todo el profesorado de una titulación sea y actúe como un equipo docente, como Rué los define (2010:33) como: 
Aquella organización de enseñantes de naturaleza colaborativa, que comparte la responsabilidad educativa sobre el mismo contingente de alumnos en un determinado tramo de la escolaridad, ya sea en un mismo nivel o curso o en un mismo ciclo educativo en un mismo establecimiento escolar.

Esto es, como un grupo de profesores que tienen un objetivo común: formar titulados en las condiciones exigidas en el Proyecto de Título, por el que van a trabajar conjunta, colaborativa y armónicamente, a fin de conseguir una docencia de calidad. Desde esta perspectiva, los Equipos Docentes, como órganos de coordinación, permitirán el éxito de la reforma universitaria, "los cambios necesarios en la docencia no son posibles sin la acción coordinada del equipo docente implicado" (Torrego y Ruiz, 2011:3).

Con el objeto de lograr la mayor eficacia se puede hablar que la labor de coordinación debe ser tanto a nivel vertical, (a lo largo de cada titulación), como horizontal, (en un mismo curso y grupo).

A nivel vertical, la coordinación hará posible que se sigan las directrices de las instancias coordinadoras (aunque estás deben tomar acuerdos de manera consensuada) y, a nivel horizontal, en las que el objetivo es la cooperación entre los docentes y la puesta en marcha del máximo intercambio de información para permitir el ajuste de la docencia a las necesidades detectadas y a las finalidades marcadas (Torrego y Ruiz, 2011:3).

La preexistencia en un mismo espacio organizativo de los dos tipos de coordinación nos permite asegurar la continuidad de los procesos de enseñanza aprendizaje y el desarrollo de las competencias. Estos tipos de coordinación deben estar establecidos formalmente con el objetivo de que el profesorado establezca relaciones, fundamentalmente formales, de cara a solucionar o prevenir problemas de distinta índole en el proceso educativo e incluso mejorarlo.

Retomando la idea inicial, la implementación de las nuevas Titulaciones al amparo de Bolonia implica un cambio estructural universitario que demanda la necesidad de coordinación en pro de una enseñanza de calidad, pero ese cambio estructural por sí sólo no hace posible el éxito de la Reforma educativa planteada, si no que además existen otros ámbitos, como ya hemos comentado, que condicionan la implantación de una cultura compartida, desde una perspectiva de cambio (Drucker, 
1993; Faure, 1993 y Escudero, 1991,1992) nos referimos a las creencias, valores, percepciones del profesorado acerca de la necesidad de coordinación. Como nos señala Prieto (2007), los profesores constituyen un grupo social cuyo quehacer docente está implicado en una tarea común, ya que es bastante improbable que ellos alcancen las metas educativas que comparten si no lo hacen a través de un esfuerzo conjunto $y$, donde las creencias, opiniones y percepciones del profesorado acerca de la necesidad de coordinación como elemento de la mejora docente juegan un papel relevante. En este sentido es frecuente encontrar a profesores que niegan que la coordinación resulte recomendable o necesaria, pues la ven como una amenaza, como un intento, por parte de la institución de limitar su margen de decisión y de adaptación y cuyos presuntos beneficios para el aprendizaje no son tales (Bain, 2007).

Asimismo, es frecuente, aunque cada vez menos encontrarse, profesionales que mantienen sus propios criterios y dinámicas de trabajo, sus propias concepciones y creencias en materia de currículo, enseñanza, aprendizaje, evaluación, disciplina... que acarrea modos paralelos y recíprocamente diferentes de enfocar los problemas y actuaciones profesionales (Hargreaves, 1991, 1996; Hargreaves y McMillan, 1995; Hargreaves y Fullan, 1997). Este ámbito puede hacer que se desarrolle una coordinación fantasma o una falsa coordinación, puede ser que formalmente se asista a las reuniones, se participe... las exigencias formales, pero en lo cotidiano y en el aula se pongan de manifiesto actuaciones aisladas o de escasa coordinación. Este ámbito saturado de componentes valorativos, personales y condicionado por la presencia del individualismo, las relaciones burocratizadas y de la inconexión entre instancias diversas (Torrego y Ruiz, 2011) constituye uno de los retos más importantes del cambio por lo que se refiere no sólo al cambio en la necesidad de coordinación sino también en la concepción del nuevo paradigma educativo.

Impregnado del ámbito anterior y relacionado con la estructura, nos encontramos el ámbito de las relaciones entre el profesorado. Actualmente, la estructura universitaria, en cada Titulación, se encuentra organizada en torno a los Departamentos y/o áreas de conocimiento que determinan $y$, a veces condicionan, las relaciones entre el profesorado. En los Departamentos se integran y desarrollan redes múltiples de relaciones tanto formales como informales que delimitan la coordinación del profesorado y evidentemente el funcionamiento de la institución. Ambas dimensiones 
-lo formal y lo informal- dan vida a la "organización real". Las relaciones formales vienen definidas por la estructura y las relaciones de carácter no formal se establecen entre los individuos y grupos identificables en la organización, más allá de aquellas que están establecidas formalmente. Pero ¿qué ocurre realmente en los Departamentos? Sin ánimo de profundizar en un análisis acerca su funcionamiento, podemos señalar siguiendo a Zabalza que los "departamentos no desarrollan una función coordinadora efectiva" (Zabalza 2000:49), nos encontramos en un momento de construcción de dinámicas de coordinación y trabajo conjunto entre los docentes para aprender, clarificar, acordar y coordinar tanto la enseñanza como el aprendizaje de las distintas asignaturas y el suyo propio y, hacer realidad aquella expectativa que se había puesto en los Departamentos como modelo de organización nuevo y más dinámico, (Zabalza, 2000) de la docencia. Hay una apuesta por la idea de grupo que funciona coordinadamente y que necesita formación para su puesta en marcha, ya que la idea de desarrollo del currículo no es estática, sino que es renovadora y adaptada continuamente a los nuevos tiempos y momentos del desarroIlo social, en lo que se denomina relación y coordinación entre la Universidad y la sociedad. En este sentido, los grupos o equipos de trabajo docentes se consideran como las unidades básicas de la organización del trabajo. Esta cooperación profesional desarrolla básicamente un trabajo en común de actividades cuyo lema es "trabajar juntos para mejorar", y, se podría añadir para aprender, ya que ésta se considera una vía idónea para el desarrollo profesional (McLaughlin y Talbert, 2001: 54). Mayor y Sánchez (1999: 173) definen los equipos docentes como "unidades de trabajo colaborativo entre profesores, donde el eje fundamental es el análisis y la reflexión sobre la práctica docente"

Sin embargo la coordinación docente también se puede sustentar en otras relaciones de de tipo no formal, el profesorado se puede coordinar más allá de lo formalmente establecido, creando nuevas finalidades y formas de aprendizaje y actividad cooperativa, creando, a la postre, comunidades de aprendizaje docente, lo que supone un gran reto para el profesorado y el establecimiento de vínculos no formales entre ellos que confluyan en el aprendizaje docente, el desarrollo profesional y organizativo y, por ende, en la mejora de la educación. En este sentido la coordinación es el cauce a través del cual "estructurar" una nueva forma de relación profesional basada en el intercambio, la responsabilidad mutua y el compromiso. 
Todos los ámbitos anteriores, cómo están configuradas las titulaciones, qué piensan los profesores acerca de las metodologías activas, la coordinación, las relaciones... tienen su repercusión en el aula. El profesor se encuentra, por un lado, con un cambio de paradigma que enfatiza los procesos de aprendizaje y acentúa el trabajo autónomo del alumnado, o lo que es lo mismo la carga de trabajo no presencial (Díez, García, e IPDDA, 2010; Tous y Amoros, 2007) y, por otro lado, en el nuevo marco europeo se enfatiza la enseñanza y aprendizaje de unos nuevos "saberes" Ilamados competencias, desde la finalidad de formar profesionales capaces de saber hacer en la práctica de diferentes contextos y saber hacerlo atendiendo a conocimientos, normas y valores validadas socialmente. Como señalan Palmer y cols., (2009) el aprendizaje por competencias no se orienta al mero desempeño inmediato de habilidades, sino que contempla la educación integral del estudiante, pues aborda tanto los conocimientos teóricos como las habilidades o conocimientos prácticos, así como las actitudes o compromisos personales que van del saber y saber hacer al saber ser o estar (Morín, 1999). Todo ello demanda un cambio sustancial en la forma de abordar los procesos de enseñanza, no sólo implica aprender "de otro modo" sino "enseñar de otro modo". Este modelo competencial (García, 2012) pretende sustituir a una enseñanza por tradición centrada en la transmisión y fragmentación de conocimientos así como, en la individualización de la enseñanza, a un modelo donde es imprescindible la colaboración de todo el profesorado para la adquisición de las competencias por parte de todos los estudiantes (Torrego y Ruiz, 2011).

A partir de todo lo anteriormente expuesto, podemos indicar los retos que plantea el nuevo modelo educativo universitario y se pueden resumir del modo siguiente:

- La organización de las titulaciones en conjuntos de enseñanzas conectadas entre sí conducentes a la adquisición de unas competencias (genéricas y específicas) exige la coordinación de la docencia a varios niveles. Desde un primer nivel, la coordinación de una asignatura impartida por varios profesores hasta coordinadores de materia, cursos, ciclos, titulaciones, facultades y universidad (Villa, 2006). Ello implica la necesidad de coordinación tanto vertical como horizontal entre los docentes y la puesta en marcha del máximo intercambio de información para permitir el ajuste de la docencia a las necesidades detectadas y a las finalidades mar- 
cadas para lograr la mayor eficacia en la labor de coordinación (Torrego y Ruiz, 2011).

- Asumir un modelo educativo formativo, integrador y constructivo que integre la visión académica, la profesional y la vital (Barnett, 2001), multi y transdisciplinar, lejos de la cultura universitaria tradicional que ofrece a los estudiantes una visión fragmentada y parcializada de la realidad. La meta será formar a los estudiantes no sólo en el conocimiento de las diferentes disciplinas, sino también en la comprensión de las mismas que les permita vivir en sociedad, de acuerdo con las expectativas que se esperan de ellos a nivel profesional. Llegar a ser profesionales reflexivos, creativos y con una sólida base de conocimientos científicos y técnicos (Martínez y Fernández, 2005). Ello implica un proyecto formativo común para definir los "perfiles profesionales" de cada título o de cada especialidad, para ello, el profesorado de cada curso, de cada titulación, tiene que coordinarse con sus compañeros (De la Calle, 2004).

- Asumir un modo de enseñar que integre la teoría-práctica, buscando espacios curriculares de integración y metodologías de aprendizaje y enseñanza que propicien un acercamiento a la realidad profesional como vía para conseguir un aprendizaje significativo, profundo y constructivo, que les permita seguir aprendiendo de manera permanente, porque habrán adquirido las estructuras mentales necesarias para afrontar nuevos y complejos problemas.

Un reto de tal envergadura precisa de la buena colaboración y coordinación entre docentes. Precisa, por tanto, de buenas relaciones profesionales tanto en el plano formal como en el no formal, de tal manera que sea posible desarrollar y asumir trabajos conjuntos y responsabilidades docentes compartidas basadas en la coordinación del profesorado.

\section{Líneas de trabajo actuales sobre la coordinación}

Las líneas de trabajo actuales sobre coordinación en el panorama universitario español se pueden enfocar en torno a tres ámbitos: estructura, procesos de enseñanza aprendizaje y creencias de los docentes acerca de la coordinación. 
Respecto de la estructura, cabe resaltar que las universidades españolas han desarrollado una estructura formal, a través de normas legales que tienen como objetivo la ordenación y coordinación de los sistemas universitarios. De esta manera, se han creado mecanismos de coordinación por titulaciones (articulación horizontal, en un mismo curso, y vertical, entre los diferentes cursos de la misma titulación) mediante comisiones, que permiten una adecuada asignación de la carga de trabajo autónomo del alumnado, la continuidad de los aprendizajes a lo largo de los títulos de Grado y de un mismo curso, la contribución a las competencias de la titulación, esto es, una adecuada planificación temporal, que asegure la adquisición de los resultados de aprendizaje.

Para ilustrar este punto, cabe señalar que, consultados los informes de seguimiento realizados por la agencia de evaluación externa (ANECA) referida a los títulos de Grado en la Rama de Ciencias Sociales y Jurídicas, sin hacer mención a la titulación y Universidad valorada aparece como recomendaciones:

- "Realizar un análisis de la calidad de los mecanismos y procedimientos de coordinación docente [...]. Se recomienda hacer un seguimiento de los mecanismos de coordinación docente que incluya un análisis valorativo cuyo objeto sea la identificación de acciones de mejora de la calidad de la titulación".

- "Revisar la optatividad para evitar reiteraciones en los contenidos con asignaturas obligatorias".

- "Optimizar la labor de los coordinadores de curso, de grupo, etc...tomar conciencia de su importancia y ajustar la labor a desarrollar por los mismos".

Destacar que son escasas las alegaciones y recomendaciones que referentes a la coordinación se han encontrado en estos informes, esto nos indica que nos encontramos en un momento de consolidación, a nivel formal, de los mecanismos de coordinación y definición de las diferentes figuras de coordinación (titulación, curso, asignatura).

Referente a la línea de trabajo centrada en los procesos de enseñanza aprendizaje, se presenta como una alternativa de cara a producir cambios valiosos en la forma de sentir, hacer y relacionarse a nivel docente de cara a la innovación y al cambio en el aula, abriendo caminos para 
el trabajo en equipo o la actividad cooperativa del profesorado con diferentes finalidades.

La progresiva introducción de esta nueva cultura compartida, a partir de las experiencias pilotos contempladas en los proyectos de Innovación Educativa, desarrolladas por las universidades en el proceso implementación de las nuevas titulaciones, afecta al trabajo del docente e introduce cambios en sus dinámicas y prácticas habituales y cotidianas. El proceso de coordinación impulsa la colaboración del profesorado en actividades conjuntas, una cultura de colaboración que introduce cambios profesionales. Por otro lado, desde esta perspectiva de la concepción del conocimiento como un "constructo" abierto a la formación del alumnado universitario que ha de favorecer un aprendizaje flexible, si bien regido por criterios fiables y justificados, que plantea una visión del conocimiento como proceso constructivo donde coordinación y planificación se presentan como procesos fundamentales de cara a orientar y articular el proceso educativo en el desarrollo del aprendizaje competencial (Quintanilla, 1998), y permite vincular el aprendizaje con la propia investigación científica, nos encontramos con numerosas experiencias de coordinación docente para el desarrollo de trabajos y proyectos interdisciplinares (Pou, Ochando y García, 2008; Monreal y Terrón, 2009; Pacheco, La Rubia y Sánchez, 2009; Pou, 2009; Diz y Jurado, 2009; Sánchez et. Al, 2009; Pozuelos, Rodríguez y Travé, 2010). Este tipo de experiencias y dinámicas, tal y como señalan Wall y Shankar, (2008), ponen de manifiesto la necesidad de asumir, por parte del profesorado, un compromiso y un apoyo mutuo de cara a sostener estas iniciativas y mejorar las prácticas educativas, ya que fomentan el aprendizaje activo y colaborativo por parte de los estudiantes y resultan el modo de enseñanza ideal para el desarrollo de competencias trasversales y/o genéricas.

También es cierto y, oídas las voces de los coordinadores Bolarín y Moreno (en prensa) que nos encontramos con algunos obstáculos que dificultan esta tarea, en primer lugar, la ausencia de reconocimientos e incentivos, lo que supone una sobrecarga de trabajo, las actividades de coordinación no se encuentran integradas en la actividad docente y ello condiciona la implementación de tareas interdisciplinares y el trabajo en equipo, unido al hecho de que este sistema esté fundamentalmente orientado a la investigación eleva el coste de oportunidad del tiempo dedicado a la docencia y lo desincentiva. Además nos encontramos con 
un segundo obstáculo que reside en las reticencias del profesor a mostrar al resto de compañeros de grupo sus métodos docentes y la sensación de tener que demostrar su profesionalidad ante el resto de profesores. Ello va unido al individualismo que caracteriza el trabajo de la mayor parte de los docentes, fomentado por las múltiples exigencias burocráticas y las desmesuradas demandas que se hacen a la carrera docente en otros ámbitos (gestión e investigación), para poder mantener la situación laboral, que con frecuencia incitan a dejar las innovaciones docentes en un segundo plano (Bolarin, Moreno y Porto, 2013).

A estas alturas, nadie pone en duda la importancia valor que tienen las creencias, las opiniones y las percepciones del profesorado universitario, de cara a superar los obstáculos y apostar por la coordinación como parte de una organización, de enseñar al alumnado compartiendo experiencias y vivencias con el resto de compañeros, es decir, de su necesidad y capacidad de pertenencia a un grupo docente (Hoy y Miskel, 1996). Es necesario ahondar en el impacto que estas creencias puedan tener en el desarrollo de una cultura compartida con el fin de mejorara su bienestar $y$, en consecuencia, su desempeño profesional el cual redundará en el rendimiento de sus estudiantes y en la calidad educativa universitaria. En esta línea se han desarrollado proyectos destinados a articular la coordinación en los diferentes Grados y, acciones formativas destinadas a generar espacios para la coordinación, para compartir experiencias con la finalidad de fomentar la necesidad de colaboración y cooperación en la consecución de una meta común, por poner un ejemplo, en la Universidad de Murcia, el Centro de Formación y Desarrollo Profesional a través del Plan de formación por Centros aborda temáticas que afectan a todos los centros universitarios, así en 2012, puso en marcha un programa de formación e intercambio de experiencias y buenas prácticas cuyo eje central eran los proyectos de coordinación.

En esta misma línea es frecuente encontrar actuaciones de formación específica para dar respuesta a las demandas y necesidades del profesorado que se encuentra en un proceso de cambio. En este sentido se han desarrollado cursos acerca de la "coordinación horizontal y vertical" Universidad de Murcia (2013); "planificación del trabajo en equipos docentes" Universidad de Alicante (2009), "coordinación para el desarroIlo de las Asignaturas", Universidad Europea Miguel de Cervantes de Valladolid (2008); "coordinación docente y proyectos interdisciplinarios" 
y seminarios de Trabajo en equipo, Universidad de Castilla-La Mancha (2011), etc.

\section{Conclusiones}

La coordinación aparece en el nuevo marco educativo, en el Sistema de Garantía de la Calidad y, en los centros como un elemento clave en la articulación de las nuevas titulaciones. A nivel educativo, constituye el elemento principal del proceso de enseñanza aprendizaje por competencias ya que, para la consecución de las mismas, es imprescindible la colaboración de todo el profesorado y, debe entenderse como una tarea colectiva para trabajar conjuntamente en el desarrollo de competencias transversales de la titulación; la formación en metodologías activas que fomenten el aprendizaje de competencias transversales y específicas. En este mismo sentido, la implantación de un modelo de enseñanza que acentúa el trabajo autónomo del alumno requiere una relación coherente y fructífera del profesorado de curso y de toda titulación para unificar y coordinar criterios en el volumen total de trabajo exigido al estudiante y, en la adecuada distribución temporal del mismo. Para ello, se hace necesario que el clima general del centro y su organización estén orientados de tal manera que favorezcan el aprendizaje de los estudiantes, convertido en el eje del centro universitario. La participación ayuda a evitar incoherencias y asumir un proyecto como propio, con la implicación de todas las personas a las que afecta, de tal manera que todo el profesorado comparta la meta global de la formación (Torrego y Ruiz, 2011).

Esa cultura basada en la consideración de la docencia y el desarrollo del proceso educativo como responsabilidad compartida (Little, 1993; Lieberman, 1995; Hargreaves, 1996) supone superar las barreras arraigadas en los supuestos tradicionales de la enseñanza y el aprendizaje para construir una cultura escolar colaborativa con creencias compartidas (Burns, 1995). Los cambios de índole cultural tienen que ver con las formas de sentir, ver, pensar y actuar. Supone, por ello, asumir y poner en práctica relaciones profesionales que apunten al intercambio y a la cooperación, crear nuevos hábitos intelectuales que nos permitan considerar diferentes puntos de vista, abandonar la cómoda rutina de las asignaturas endogámicas, la incomunicación de los 
docentes y la disociación entre la teoría y la práctica (Ruíz, Castaño y Boronta, 1999).

El panorama actual nos sitúa en un momento de construcción y consolidación de estructuras formales que suponen el "andamiaje" sobre el que asentar este cambio organizativo. Pero, también es cierto que una vez superada esta etapa de definición y organización de la nueva estructura universitaria, los datos referidos al desarrollo de trabajos interdisciplinares $y$, por tanto, de coordinación han quedado reducidas al "boom" producido por las experiencias piloto, de la misma manera que los cursos de formación que fomentan el intercambio de experiencias y donde los obstáculos parecen el eje central que favorecen que nuestros centros universitarios no se encaminen hacia la conversión en comunidades de aprendizaje. En el panorama actual de nuestras universidades la coordinación parece una utopía, aún queda mucho por hacer.

\section{Referencias bibliograficas}

Bain, K. (2007). Lo que hacen los mejores profesores universitarios. (2 ${ }^{\mathrm{a}}$ edición). Valencia: Universidad de Valencia.

Bandura, A. (Ed.) (2004). Autoeficacia: cómo afrontamos los cambios de la sociedad actual. Bilbao: Desclée de Brouwer.

Barnett, R. (2001). Los límites de la competencia. El conocimiento, la educación superior y la sociedad. Barcelona. Gedisa.

Bolarín, M. J. Moreno, M. A y Porto, M. (2013). La interdisciplinariedad y su contribución a la adquisición de las competencias: las voces del alumnado. Revista de Docencia Universitaria. Vol.11 (2), (pp. 443-462)

Bolarin, M.J., Moreno, M.A., (en prensa). La coordinación docente en la universidad: retos y problemas a partir de Bolonia. Profesorado:Revista de Currículum y Formación del profesorado.

Burns, B. (1995). Paradigms for research in teaching. En L. W. Anderson, (Ed.). The International Encyclopaedia of Teaching and Teacher Education (2nd ed.), (pp.17-20). Oxford: Pergamon.

Calderón, C. y Escalera, G. (2008). La evaluación de la docencia ante el reto del Espacio Europeo de Educación Superior (EEES). Educación XXI, 11, (pp. 237-256).

Díez, M. C., García, J. N., e IPDDA. (2010). Percepción de metodologías docentes y desarrollo de competencias al EEES. Boletín de Psicología, 99, (pp.45-69).

De la Calle, M. J. (2004). El reto de ser profesor en el contexto de la convergencia europea. La formación pedagógica como necesidad. Revista Interuniversitaria de Formación del Profesorado, 18 (3), (pp. 251-258).

Diz, J. y Jurado, M. (2009). Actividades coordinadas entre bioestadística y zoología en 
primer curso de la Licenciatura en Ciencias Biológicas. Recuperado de http://congresos.um.es/redu/2-09paper/view/1801/1771.

Drucker, P. (1993). Cultura de las organizaciones. Administración y futuro de los '90 en adelante. Buenos Aires: Sudamericana.

Escudero, J. M. (1991). La formación centrada en la escuela. En J. López Yañez, (Coord.). El Centro Escolar, (pp.7-36). Universidad de Sevilla.

Escudero, J. M. (1992). Innovación y desarrollo organizativo de los centros escolares. Ponencia presentada al // Congreso Interuniversitario de Organización Escolar, Sevilla, España.

Escudero, J. M. (2009). Las competencias profesionales y la formación universitaria: posibilidades y riesgos. Revista interuniversitaria de pedagogía social, 16, (pp.65-82).

Faure, G. (1993): "Estructura, organización y eficacia de la empresa: fundamentos de gestión directiva". Deusto, DL, Madrid.

Fernández A. (2006). Metodologías activas para la formación de competencias. Educatio siglo XXI, 24, (pp.35 -56).

García, M. P. (2012). Aprendizaje y evaluación de competencias. Curso de formación: Aprendizaje y evaluación de competencias. Documento inédito. Centro de Formación y Desarrollo Profesional. Universidad de Murcia.

González, Ma T; Nieto, J. M. y Portela, A. (2007). Organización y gestión de centros escolares: dimensiones y procesos. Madrid: Pearson Educación.

Hargreaves, A. (1991). Cultures of Teaching: a focus for changes. En A. Hargreaves y M. Fullan, (Ed.). Understanding Teacher Development (pp. 216-240). New York: Teacher's College Press y Cassell.

Hargreaves, A. (1996). Profesorado, cultura y postmodernidad (Cambian los tiempos, cambia el profesorado). Madrid: Morata.

Hargreaves, A. y McMillan, R. (1995). The balkanization of Secondary School teaching. En L. S. Siskin y J. W. Litle, (Eds.). The Subject in Question. Departmental organization and the High School, (pp.141-171). Londres: Theacher College Press.

Hargreaves, A. y Fullan, M. (1997). What's worth fightin for out there? New York: Teachers Collage.

Hoy, W. y Miskel, C. (1996). Educational administration: Theory, research, and practice. New York: McGraw-Hill.

Little, J. (1993). Professional community in comprehensive high school: the two worlds of academic and vocational teacher. En J. Little y W. McLaughhlin, (Eds.). Teacher's work. Individuals, colleagues an context, (pp.137-164). New York: Teacher Collage Press.

Ley Orgánica 4/2007, de 12 de abril, por la que se modifica la Ley Orgánica /2001, de 21 de diciembre, de Universidades, publicado en BOE núm. 89 de 13 abril 2007.

Lieberman, A. (1995). The work of restructuring School: building fron the gruond up. New York: Teachers College Press.

Mayor, C. y Sánchez, M. (1999). Los equipos docentes: una contribución formativa a la calidad del profesorado universitario. XXI. Revista de Educación, 1, (pp.157-176).

Martínez, B. y Fernández, A. (2005). El cambio de cultura docente y el Espacio Europeo de Educación Superior. En V. Esteban Chapapría (Ed.). El Espacio Europeo de Educación Superior 95-163. Valencia: Instituto de Ciencias de la Educación. 
La coordinación docente en la universidad: análisis de la situación actual

Mclaughlin, M. y Talbert, J. (2001). Professional communities and the work of high school teaching. Chicago: University of Chicago Press.

Ministerio de Educación y Ciencia (1992). La formación del profesorado universitario. Madrid: Ministerio de Educación y Ciencia.

Monreal Gimeno, M. C. y Terrón Caro, M. T. (2009). Una experiencia interdisciplinar en Ciencias Sociales en la Universidad Pablo de Olavide. En Fargueta Cerdá, Fernández March y Maiques March (Eds.). V Congreso Iberoamericano de Docencia Universitaria. Enseñar y aprender en la universidad del s. XXI: propuestas y condiciones. Valencia: Editorial de la Universidad Politécnica de Valencia, CD.

Morin, E. (1999). Los siete saberes necesarios para la educación del futuro. París: UNESCO.

Pacheco Reyes, R.; La Rubia García, M. D. y Sánchez Villasclaras, S. (2009). Una experiencia interdisciplinar: estudio de la corrosión en nuestro entorno a través de imágenes. En Actas de la IV Reunión Innovación Docente en Química, INDOQUIM. Burgos: Servicio de Publicaciones de la Universidad de Burgos, CD.

Palmer, A., Montaño J. J., y Palou, M. (2009). Las competencias genéricas en la Educación superior. Estudio comparativo entre la opinión de empleadores y académicos. Psicothema, 21 (3), (pp.433-438).

Perrenoud, P. (2002). Construir competencias desde la Escuela. Santiago de Chile: Dolmen Ediciones.

Pou, R., Ochando, L., García, R., y Bertomeu, J. R. (2008). Metodologías activas en el marco del EEES: aprendizaje cooperativo basado en trabajos en equipos coordinados. Anales de la Real Sociedad Española de Química, 104, 2, (pp.135-139).

Pou, R. (2009). El desarrollo de proyectos interdisciplinarios. En Seminario de reflexión RED-U-USC: La coordinación mediante equipos docentes en ES: fortalezas, recursos y necesidades. Recuprado de http://congresos.um.es/redu/2-09/paper/view/1661/1631.

Pozuelos, F., Rodríguez, F., y Travé, G (2012). El enfoque interdisciplinar en la Enseñanza universitaria y el aprendizaje basado en la investigación. Un estudio de caso en el marco de la formación Revista de Educación, 357, (pp.561-585).

Prieto, L. (2007). Autoeficacia del profesor universitario. Eficacia percibida y práctica docente. Madrid: Narcea.

Real Decreto 1509/2005, de 16 de diciembre, por el que se establece la estructura de las enseñanzas universitarias y se regulan los estudios universitarios oficiales de Grado, publicado en BOE núm. 303 de 20 diciembre 2005.

Real Decreto 861/2010, de 2 de julio, por el que se modifica el Real Decreto 1393/2007, de 29 de octubre, por el que se establece la ordenación de las enseñanzas universitarias oficiales, publicado en BOE núm. 161 de 3 julio 2010.

Quintanilla, M. A. (1998). El reto de la calidad en las universidades. En J. Porta y M. Llanodosa (Coords.). La universidad en el cambio de siglo, (pp.79-101). Madrid: Alianza.

Rué, J. y Lodeiro L. (Eds.). (2010). Equipos Docentes y nuevas Identidades Académicas. Madrid: Narcea

Ruíz, E., Castaño, N., y Boronta, J. (1999). Reflexiones sobre el enfoque interdisciplinar y su proyección práctica en la formación del profesorado. En Revista Electrónica Interuniversitaria de Formación del Profesorado, 2 (1).

Sánchez, M. T., Fraile, E., Frechoso, J.C., Bachiller, J. M., Carrera J. y Zarandona J. M. 
(2009). Experiencias de colaboración a través de proyectos interdisciplinarios. Revista d'Innovació Educativa, 3, (pp.52-61).

Schein E. (1988). La cultura empresarial y el liderazgo. Barcelona: Plaza y Janés.

Thomas, M. y lon, G. (2008). Contrastando dos modelos de análisis de la cultura organizativa de la universidad. Bordón, 60, (pp.131-147).

Tomàs, C., Armengol, A., Borrell, A., Castro, D., Esteve, J.; Feixas, M., Gairín, J., y Marquès, P. (2001). El cambio de cultura en las universidades del siglo XXI. Recuperado de http://diposit.ub.edu/dspace/bitstream/2445/22320/1/508145.pdf

Torrego, L. y Ruiz, C. (2011). La coordinación docente en la implantación de los títulos de Grado. REIFOP, 14 (4). Recuperado de http//www.aufop.com.

Tous, C, Amorós, A. (2007). Motivaciones para el estudio en universitarios. Anales de psicología. 23, 1 (pp.17-24)

Villa, A. (2006). El proceso de convergencia europeo y el papel del profesorado. Foro de educación, 7, (pp.103-117).

Wall, S. y Shankar I. (2008). Adventures in Transdisciplinary Learning. Studies in Higher Education, 5, 33, (pp.551-565).

Zabalza, M. (2000). El papel de los departamentos universitarios en la mejora de la calidad de la docencia. Revista Interuniversitaria de Formación del Profesorado, 38, (pp.47-66).

Zabalza, M. (2004). Guía para la planificación didáctica de la docencia universitaria en el Marco del EEES. Documento de trabajo. Recuperado de http://www.upcomillas.es/ eees/Documentos/guiaplan.pdf. 
